\title{
High-Resolution Tracking of Single-Molecule Diffusion in Membranes by Confocalized and Spatially Differentiated Fluorescence Photon Stream Recording
}

\author{
Steffen J. Sahl, ${ }^{[\mathrm{b}]}$ Marcel Leutenegger, ${ }^{[\mathrm{c}]}$ Stefan W. Hell, ${ }^{[\mathrm{[a]}}$ and Christian Eggeling*[d]
}

\begin{abstract}
The performance of a method is assessed which allows for the spatiotemporal tracking of single dye-labeled molecules during two-dimensional (2D) diffusional transits through the focal area of a modified confocal microscope. In addition to facilitating the observation of molecular diffusion paths at the shotnoise limit of bright organic emitters with spatial and temporal precisions of $\sim 10-20 \mathrm{~nm}$ and $<0.5 \mathrm{~ms}$, respectively, the direct access to the complete stream of detected photons is beneficial for characterizing nanoscale details such as transient pausing (binding). We discuss technical aspects of this approach, along with results from its application to measuring lipid mem-
\end{abstract}

brane dynamics in live mammalian cells. Presented topics in clude a discussion of the advantages of the single-photon collection mode and instrument as well as computational considerations for the localization process. A proof-of-principle experiment shows that optical nanoscopy by stochastic singlemolecule switching and position readout could be implementable in parallel with such fast molecular tracking. This would allow direct access to contextual imaging data of local cytoskeletal structural elements or localized longer-lived protein as semblies.

\section{Introduction}

The measurement of lateral diffusion characteristics for many cellular plasma membrane components poses severe challenges to experimental design. For instance, lipids and small proteins have considerable mobility, displaying effective macroscopic diffusion coefficients of $0.1-1 \mu \mathrm{m}^{2} \mathrm{~s}^{-1}$, as inferred by state-of-the-art methods. ${ }^{[1-10,27]}$ While these molecules diffuse rapidly, their average mobility may be quantified by techniques such as fluorescence recovery after photobleaching (FRAP), ${ }^{[3-5]}$ confocal (scanning) fluorescence correlation spectroscopy $(F C S)^{[6-10]}$ or image correlation spectroscopy ${ }^{[11]}$ in conjunction with suitable molecular probes (labels) for the molecules under investigation. Owing to the limited spatial and/or temporal resolution of these approaches, a "global" or "macroscopic" diffusion coefficient is measured, that is, a scalar value reflecting the overall mobility over many- $\mu \mathrm{m}^{2}$ areas. Crucially, these methods only indirectly detect different populations of mole-

\footnotetext{
[a] Prof. S. W. Hell

Department of NanoBiophotonics

Max Planck Institute for Biophysical Chemistry

37077 Göttingen (Germany)

E-mail: shell@gwdg.de

[b] Dr. S. J. Sahl

Department of Chemistry, Stanford University Stanford, CA 94305 (USA)

[c] Dr. M. Leutenegger

École Polytechnique Fédérale de Lausanne

1015 Lausanne (Switzerland)

[d] Dr. C. Eggeling

MRC Human Immunology Unit

Weatherall Institute of Molecular Medicine

University of Oxford, Oxford OX3 9DU (UK)

E-mail: christian.eggeling@rdm.ox.ac.uk
}

cules (slow ones versus fast ones for instance) and often fail to reveal alternations between fast and slow motions of one and the same molecule. In other words, details in the diffusive trajectories of molecules such as transient binding (and thus pausing) events relative to the frame of reference of the surrounding molecules are often not detected. Such short-lived events are hard to capture because the spatial length scales of interest $(\ll 250 \mathrm{~nm})$ are inaccessible to diffraction-limited approaches like FRAP and confocal FCS.

Performing FCS in nanoscale observation areas defined by diffraction-unlimited stimulated emission depletion (STED) microscopy, recent studies ${ }^{[12-16]}$ directly revealed details in the highly dynamical lipid membrane diffusion far below the optical diffraction limit. STED-FCS will likely play major roles in elucidating cell membrane organization at down to molecular spatial and $\sim$ ms temporal scales. ${ }^{[17]}$ These intricacies in the transient associations of all components of the two-dimensional fluid plasma membrane likely confer larger-scale structure and organize the membrane laterally to perform many cellular functions. ${ }^{[18-21]}$

Single-particle and single-molecule tracking approaches provide a complementary strategy to study membrane molecule diffusion (see, for example, refs. [19,20,22-29]). In principle, the spatial limitation can be largely overcome since localization of isolated fluorescence (or other) emitters is possible to high statistical confidence down to the few-nanometer range by finding the center of an adequately sampled photon distribution on the detector, either in wide-field area detection or in a confocal scanning geometry. ${ }^{[30,31]}$ Even swift molecular movements can be traced optically if sufficient signal is obtained from the single molecule or particle in question-bright labels 
such as gold colloids or quantum dots have often been employed $^{[19,20,29]}$ —and provided that sensitivity and time response of the detection elements are adequate.

The authors recently demonstrated the application of a dedicated instrument to the tracking challenge of fast lipid diffusion in living mammalian cells. ${ }^{[32]}$ Plasma membrane lipids were followed with high spatial precision $(\sigma \approx 10-20 \mathrm{~nm})$ and temporal resolutions of $<0.5 \mathrm{~ms}$ for the bright dye Atto647N conjugated to the phosphoglycerolipid phosphoetanolamine $(\mathrm{PE})$ and sphingomyelin (SM). Notably, the propensity of SM to be transiently trapped for several milliseconds correlated with the cholesterol content of the PtK2 cell plasma membrane in this study. ${ }^{[32]}$

The method is conceptually simple, and different to a conventional camera-based wide-field tracking experiment (Figure $1 \mathrm{~A}$ ), where fitting of the single-molecule image on the detector in a sequence of camera frames provides a position estimate at defined time intervals given by the camera frame rate. In contrast, in fast confocalized tracking ${ }^{[32]}$ (Figure 1B), we recorded sparsely labeled single molecules as they diffused oneby-one through a confocal excitation spot. The fluorescence was focused onto an arrangement of three detection pinholes rather than one. For this purpose, a multi-mode fiber bundle coupled to single-photon detectors and counting electronics was used (Figure 1C,D). The relative signals detected in the three overlapping detection volumes provided nanoscale position information and could be continuously read out, thus providing an effective time resolution which is limited on the instrument side merely by the nanosecond response time of the single-photon detectors.

We note that our method is not limited to fluorescence as the signal-giving mechanism, but that triangulation is compatible with scattering or other signal. A bigger membrane sample area could be covered by using larger numbers of point detectors in suitable spatial arrangements. A key advantage of our scheme is the flexible trade-off between spatial and temporal resolution as discussed in Section 2.4.4. In this Article, we discuss several points pertaining to our method from an implementation and application standpoint.

\section{Results and Discussion}

Our method relies on: 1) the identification of single-molecule transits through our diffraction-limited observation spot, and 2) the determination of their spatiotemporal tracks within the observation area by comparing the signal triple $\vec{S}(t)=\left(S_{1}, S_{2}, S_{3}\right)$ detected on each of the three detectors with a calibration map $\left\{\vec{R}=\left(R_{1} R_{2} R_{3}\right)\right\}$ at different time points $t$ of the transit. We established the calibration map by scanning a photostable nanometric emitter through the focus whilst recording the relative three detector signals as a function of the emitter position. We used a $40 \mathrm{~nm}$ fluorescent bead and scanned a $500 \mathrm{~nm} \times$ $500 \mathrm{~nm}$ region at $2.5 \mathrm{~nm}$ spatial sampling. ${ }^{[32]}$

\subsection{Efficient Position Estimation by a Logarithmic Likelihood Maximization Scheme}

We discuss the process of matching counts on the three detection elements during a time interval to the experimental reference map (calibration). ${ }^{[32]}$ This is realized by an estimator of the likelihood that the signal triple $\vec{S}=\left(S_{1}, S_{2}, S_{3}\right)$ corresponds to a normalized reference triple $\vec{R}=\left(R_{1}, R_{2}, R_{3}\right)$ in the calibration map $\{\vec{R}(x, y)\}$ for different lateral coordinates $x, y$ within the observation spot. The reference triples shall be normalized throughout this discussion such that $R_{1}+R_{2}+R_{3}=1$. Maximizing the likelihood of observing $\vec{S}$ given $\vec{R}(x, y)$ yields the most likely position $(\hat{x}, \hat{y})$ of the particle in the focal plane.

The likelihood of detecting $n$ photons for $\langle n\rangle$ detected photons on average is given by the Poissonian probability density function [Eq. (1)]:

$$
\begin{aligned}
p(n,\langle n\rangle) & =\frac{\langle n\rangle^{n}}{n !} \exp (-\langle n\rangle) \\
& =\exp (n \cdot \ln (\langle n\rangle)-\ln (\Gamma(n+1))-\langle n\rangle)
\end{aligned}
$$

where $\Gamma(k)$ is the gamma function. Therefore, the likelihood of detecting $\vec{S}$ photons at a position with $N \vec{R}$ expected photons on average is given by the product of the probabilities [Eq. (2)]:

$L(\vec{S}, N \vec{R})=\prod_{i=1}^{3} p\left(S_{i}, N R_{i}\right)$

Here, the calibration triples $\vec{R}(x, y)$ are scaled to the total number of detected photons $N=S_{1}+S_{2}+S_{3}$. The most likely position for an observed triple $\vec{S}$ is then the location where this probability is maximum, which is equivalent to finding the maximum of the log likelihood, that is, of the sum [Eq. (3)]:

$$
\begin{aligned}
\ln (L) & =\sum_{i=1}^{3} \ln \left(p\left(S_{i}, N R_{i}\right)\right) \\
& =\vec{S} \cdot \ln (N \vec{R})-\sum_{i=1}^{3} \ln \left(\Gamma\left(S_{i}+1\right)\right)-N
\end{aligned}
$$

The last two terms are invariant with the position and can be neglected for finding the most likely position. Moreover, the scaling of the average triples can be skipped because it only leads to an additive term $N \ln (N)$ that is again invariant with the position. Finally, the remaining scalar products are calculated using a matrix product, that is [Eq. (4)]:

$\vec{S}(t) \cdot \ln (\vec{R}(x, y))$

which is searched for the maximum at each time $t$ to assign the most likely position $(\hat{x}, \hat{y})(t)$. It is worth noting that this scheme is readily extensible for a higher number of detection channels.

For a sequence of observations $\vec{S}(t)$ with a known photon number $N$ as obtained by partitioning the photon stream into 
A) Single particle/molecule tracking by video microscopy

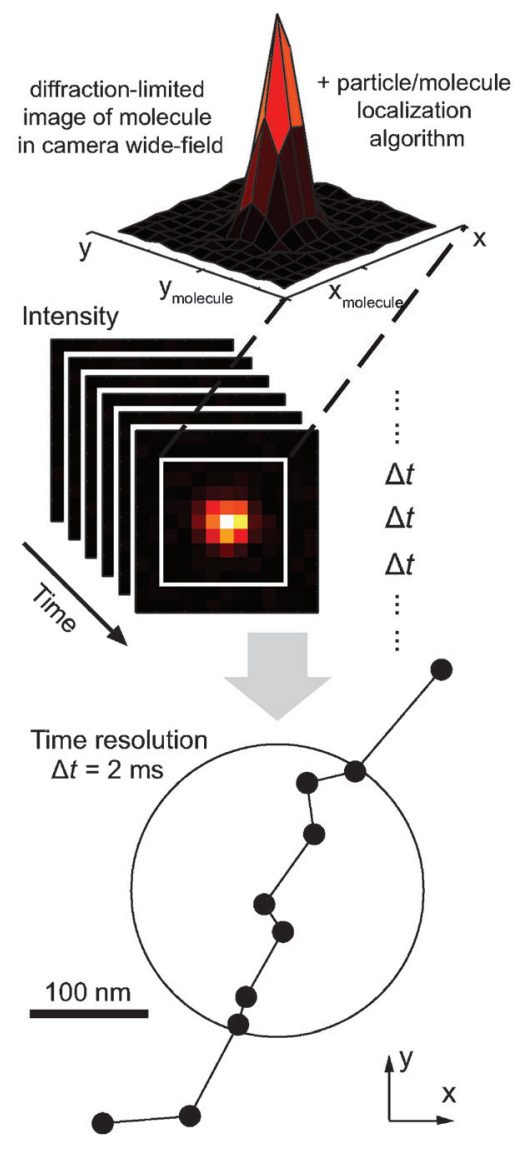

B) Fast confocalized single

800 . molecule tracking

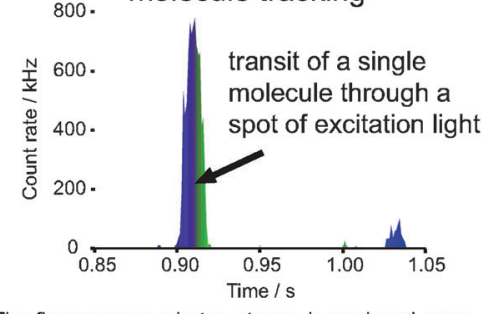

The fluorescence photon stream is analyzed over time in a spatially resolved way ( 3 point detectors in a confocalized microscope with spatially overlapping detection efficiencies)

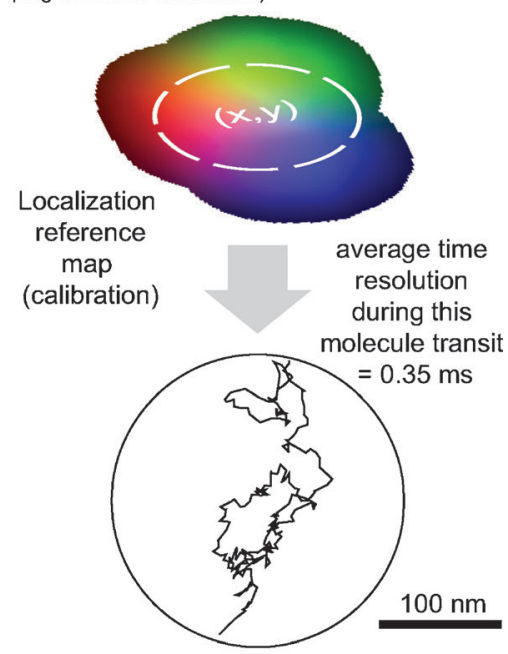

y $\quad N=250$ photon window for localization, advanced at $n=25$ photons (realistic count rate in absolute peak of $750 \mathrm{kHz}$ )

C)
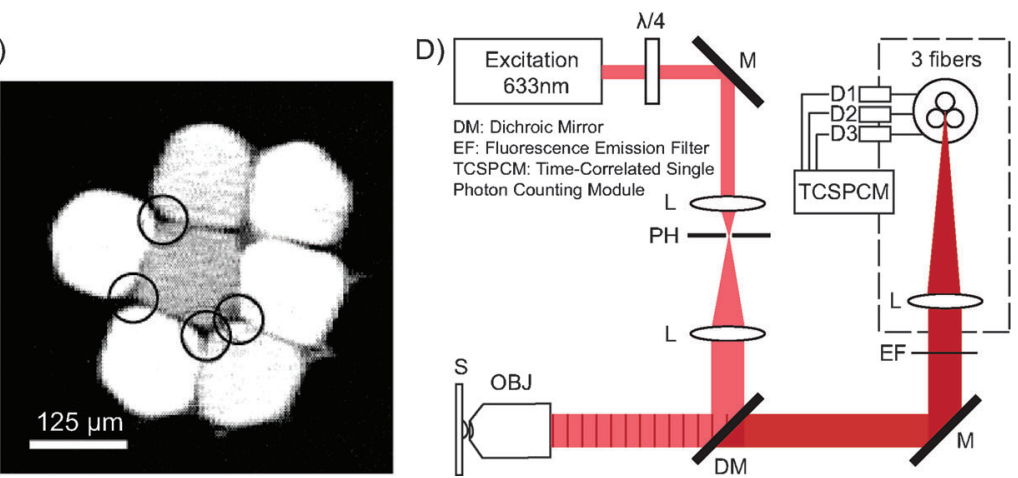

Figure 1. Tracking in a confocal microscope by spatially resolved fluorescence photon counting in contrast to tracking by camera-based video microscopy. A) In single-particle or single-molecule tracking by video microscopy, the region of interest is imaged in a wide-field microscope onto a camera. The contrastgiving mechanism is typically either scattering (from colloids) or fluorescence from single isolated molecules, whose signal is detected on a camera and the particle position recovered from the observed intensity distribution. From the image stack with time lags between images $\Delta t=\Delta t_{\text {exposure }}+\Delta t_{\text {readout }}$ a spatiotemporal trajectory may be constructed. B) Fast confocalized tracking enables single-molecule observation with high sensitivity. The elicited quasi-continuous stream of fluorescence photons from an individual molecule passing through the focus is recorded on spatially distinct detectors (fibers connected to singlephoton-counting avalanche photodiodes). Because the signal is available at the single-photon level, the molecule may be identified from a burst in the signal time trace and localized from the ratio of signal levels detected on the three different detectors in quick succession with high flexibility with respect to the signal integration. The approach achieves excellent signal-to-background ratios ( $>200)$ for small dye-labeled molecules in membranes of living cells and exceeds other published experiments with single-fluorophore labels by a factor of five to ten in spatiotemporal resolution, though at the expense of a smaller observation area. Trajectories obtained with both methods are shown. C) Micrograph of fused fiber bundle. The central fiber is used in combination with two adjacent fibers. The small circles indicate some possible combinations. The described concept of the localization reference map, i.e., the encoding of the relative detection efficiencies as a function of the emitter position, readily deals with point-like detectors in almost arbitrary configurations. The fibers need not be equidistant from each other, although this is advantageous for a uniform localization precision. D) Details of the optical setup with OBJ =objective, $\mathrm{DM}=$ dichroic mirror, $\mathrm{L}=$ lens, $\mathrm{M}=$ mirror, $\mathrm{PH}=$ pinhole, $\mathrm{TCSPCM}=$ time-correlated single photon counting module, $\mathrm{EF}=$ fluorescence emission filter, $\mathrm{D} 1-$ D3 = single-photon-counting avalanche photodiodes (APDs). 
windows of $N$ consecutive photons, the maximum likelihood estimation takes an especially simple form: the photon counts in two channels determine the triple $\vec{S}$ and thus the localization, since $S_{3}=N-S_{1}-S_{2}$. The resulting position from maximizing the likelihood may be compactly saved in a $(N+1) \times(N+2)$ matrix $R(i, j)$ (the calibrated reference map, Figure $2 \mathrm{~A}$ ). Then, the positions for triples $\vec{S}$ satisfying $S_{1}+S_{2}+$ $S_{3}=N$ can be looked up very efficiently, for instance by reading the matrix elements $\hat{x}=R\left(1+S_{1}, 1+S_{2}\right) \quad$ and $\hat{y}=R\left(N+1-S_{1}, N+2-S_{2}\right)$.
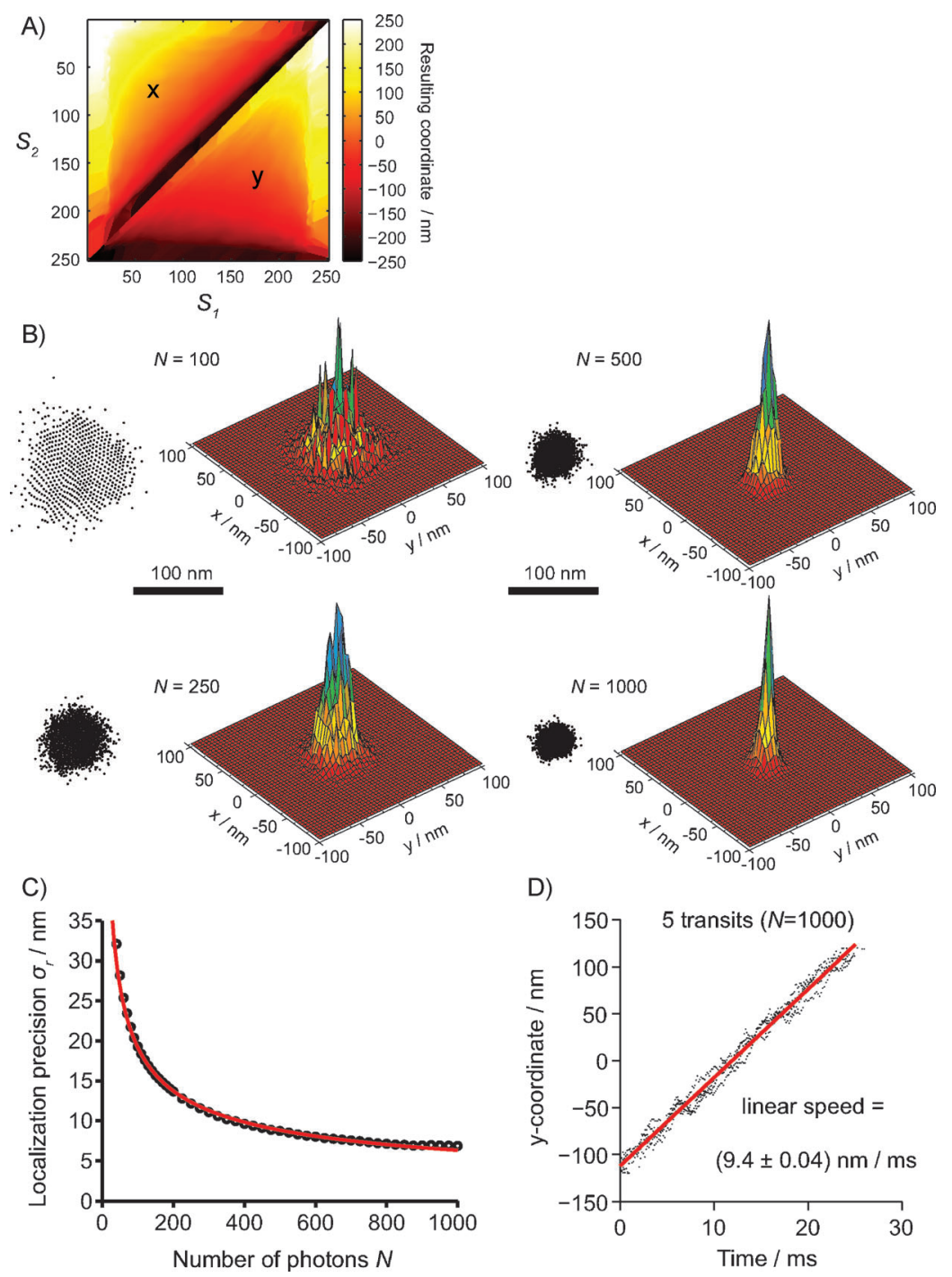

Figure 2. Maximum likelihood estimation (MLE) of position for fixed number of total photons. A) Look-up table holding the results of all possible localization outcomes for $N=250$ photons, and for different number triples $\left\{S_{1}\right.$, $S_{2}, S_{3}$ \} detected on the three different detectors. The configuration space for $N$ photons on just three detectors is dramatically reduced, and all possible combinations $\left\{S_{1}, S_{2}, S_{3}=N-S_{1}-S_{2}\right\}$ can be saved in a $2 D$ matrix of dimensions $(N+1) \times(N+2)$ storing the most likely positions $(\hat{x}, \hat{y})$. The instant localization is then immediately found by looking up the positions at the corresponding count values. This makes the matching process very fast. The plot shows resulting coordinates $(\hat{x}, \hat{y})$ as a function of $S_{1}$ and $S_{2}$. B) Localization precision $\sigma_{\mathrm{r}}$ versus photon number $N$. An isolated $24 \mathrm{~nm}$ fluorescent bead was localized repeatedly for a varying number of total photons $N$. The spread of 10,000 independent localizations in each case was examined (examples $N=100,250,500$ and 1000, Scale bar, $100 \mathrm{~nm}$ ). C) The radial standard deviation $\sigma_{\mathrm{r}}=\left(\sigma_{\mathrm{x}} \cdot \sigma_{\mathrm{y}}\right)^{1 / 2}$ was found to scale with $N^{-1 / 2}$ as expected. D) Single $24 \mathrm{~nm}$ fluorescent beads scanned on a straight line through the observation region ( 5 transits) are adequately localized. The set linear scan speed of $\sim 10 \mathrm{~nm} \mathrm{~ms}^{-1}$ was reproduced with a relative error of $-6 \%$ (settings: 100 pixels at dwell time $1 \mathrm{~ms}$ for a total distance of $1 \mu \mathrm{m}$ ).

\subsection{Localization Performance}

Depending on the number of signal photons combined in a single localization estimate, the localization precision (statisti(a) As a demonstration of instrument, we repeatedy localized isolated $24 \mathrm{~nm}$-sized fluorescent beads at rest, utilizing a varying number of photons $N$. Then the spread of 10000 independent localizations was examined in each case (Figure $2 \mathrm{~B}$ ). The radial standard deviation [Eq. (5)]:

$\sigma_{r}=\left(\sigma_{x} \cdot \sigma_{y}\right)^{1 / 2} \approx \sigma_{x} \approx \sigma_{y}$

of the cloud of localized positions is a robust measure of the statistical localization precision and was found to be $\sigma_{r}=12 \mathrm{~nm}$ for $N=250$ photons, scaling with $N^{-1 / 2}$ as is expected for shotnoise-limited behavior (Figure $2 \mathrm{C}$ ). Fluorescent beads scanned linearly through the observation region were adequately localized (Figure 2D). These virtually background-free measurements of immobilized beads on clean glass substrates represented the absolute lower bound on the localization precision attainable with our instrument.

For tracking lipids in live-cell PtK2 plasma membranes (see below), we chose well-prepared clean samples with a labeling density so low as to guarantee well-separated single-molecule transits. ${ }^{[32]}$ We observed a background count rate of $\sim 3-5 \mathrm{kHz}$ $(10 \mathrm{kHz}$ at most). The relevant figure of merit for the localization uncertainty is the signal/ background $(\mathrm{S} / \mathrm{B})$ ratio, which exceeded 50 and usually lay in the range of 100-250 for our lipid tracking without high background from internalized vesicles or such. At high $S / B$, the background is negligible compared to the signal and its effect can be ignored. However, for S/ $B \lesssim 10$, localizations will be significantly biased towards the center of the observation area, as counts $\vec{S}$ in the three channels tend to become similar to each 
other for higher, uncorrelated background. In that case, the calibration map should be recorded under comparable S/B conditions to the experiment, for example, by scanning a fluorescent bead in an extended layer of $\operatorname{dim}$ fluorophores to mimic background.

\subsection{Nanoscale Position Information is in the Gradients of Fiber Detection Efficiencies: Optimization of Parameters}

The physical separation of the detection pinholes (multimode fiber cores), the lateral magnification $M$ of the microscope, and thus the back-projected separation of the pinhole centers in the excitation focus (sample space, Figure $3 \mathrm{~A}$ ) determine the sensitivity of the relative signals (i.e. the signal fractions of the detectors) to changes in lateral position of the point emitter.
A)

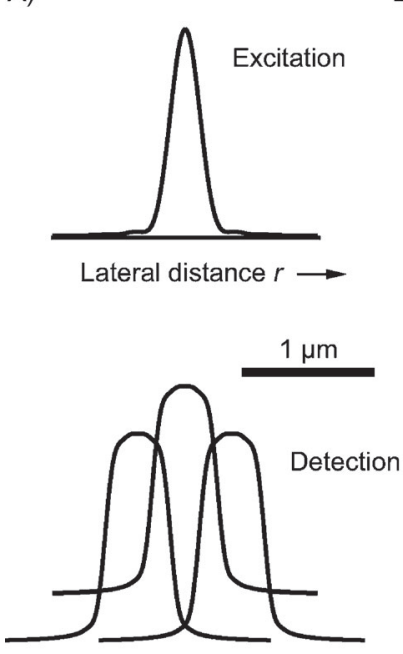

B)
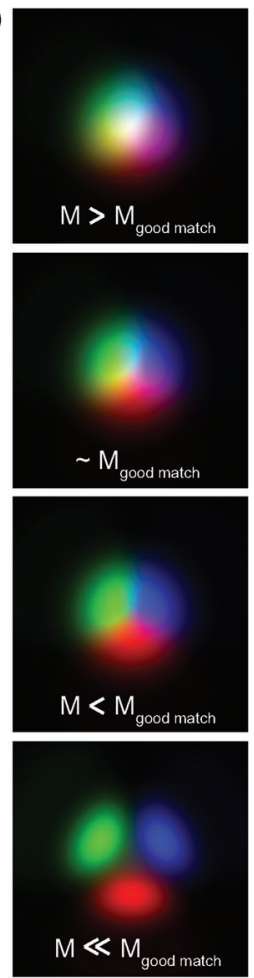

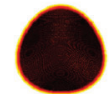

$500 \mathrm{~nm}$

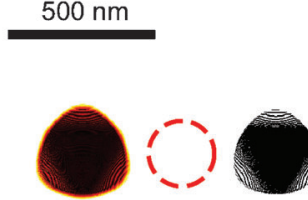

satisfies $|\Delta|<2 \mathrm{~nm}$
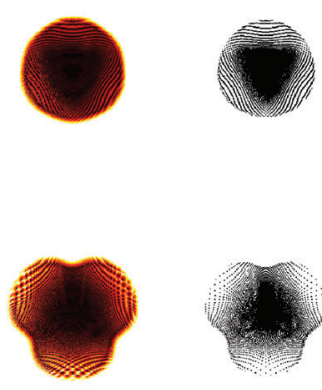

$|\Delta| / n m$

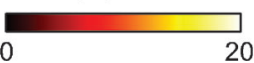

Figure 3. Optimization of spatial gradients in the relative detected photon numbers on detectors 1 to 3 . A) Projection of the three fiber input faces (detection elements) with collection efficiencies $Q_{i}(x, y)$ back into the sample space. There, the intensity distribution of the excitation light $l_{\text {exc }}(x, y)$ is given by the diffraction-limited pointspread-function (PSF). B) The lateral magnification factor $M$ of the imaging onto the fibers determines how well the projected detector separation is matched with respect to the excitation PSF (see text). A condition of a reasonable degree of confocality must also be fulfilled, allowing both high collection efficiencies and sufficient optical sectioning. A lateral magnification of $\sim 200-250 \times$ was a useful compromise for high localization precision over $\mathrm{a} \sim \varnothing 250 \mathrm{~nm}$ region. The three-color RGB plots (left column) show overlays of the signals in the three detection elements $Q_{1}-Q_{3}$, represented by red (R), green $(G)$ and blue $(B)$ for four different regimes of $M$, with the focal intensity distribution of the excitation laser $l_{\text {exc }}(x, y)$ the same in each case. $M$ determines the spacing between the detection elements as projected in sample space. A high distinguishability (uniqueness) of count triples for neighboring points $(x, y)$ within the field of view is to be achieved, that is, a richer color palette with more subtle color differences in this representation (look-up table). $|\Delta|$ is the localization error (small systematic bias) introduced in the position look-up process due to insufficient uniqueness of count triples ${ }^{[32]}$ (right columns). It is calculated by taking the count triple at any given position (pixel) from an experimental (or, here: idealized theoretical) localization reference map, localizing with this map, and comparing the localized position to the original position. Biases $|\Delta|$ of $<2 \mathrm{~nm}$ are guaranteed throughout a $250 \mathrm{~nm}$ circular area (red circle) if $M$ is chosen appropriately. Black pixels (rightmost column) have $|\Delta|<2 \mathrm{~nm}$.
Fast focus field calculations based on ref. [33] allowed us to tudy this effect and optimize the magnification. The gradients in signals tended to be highest near the overall center of the and the signal is captured mostly by one detector for most of the time. On the other hand, for $M>M_{\text {good match, the detectors }}$ refficiencies overlap too much). In both cases, the ared distinguishable count combinations becomes small. A latera magnification of $\sim 200-250 \times$ yielded an adequate coverage of the central $250 \mathrm{~nm}$ at high spatial precision. The combinations molecule in this region are unique, which is key for precise localizations. The empty space (gap between fibers at the center) is negligible, amounting to $<1 \%$ of the three fiber input faces by total area.

\subsection{Advantages of Quasi- Continuous Fluorescence Sampling}

The state-of-the-art detection and recording with time-tagged single-photon-counting electronics $^{[32]}$ allowed us to push molecule localization to the shot noise limit. Assuming low levels of background fluorescence (as are found in our sparsely labeled cell membranes), we are only limited by the number of photons detected from the emitting molecule.

\subsubsection{Enhanced Statistical Basis of Trajectories}

Experimental realizations of single-molecule tracking always represent an incomplete sampling of the true trajectory taken by the observed molecule. For instance, Figure $4 \mathrm{~A}$ shows a molecule trajectory resulting from a random walk at a diffusion constant $D=0.3 \mu \mathrm{m}^{2} \mathrm{~s}^{-1}$, simulated with a time increment of $1 \mu \mathrm{s}$. The position is plotted every $50 \mu$ s for clarity of display. In the following we assume illumination conditions concomitant with a conservatively chosen 
A)

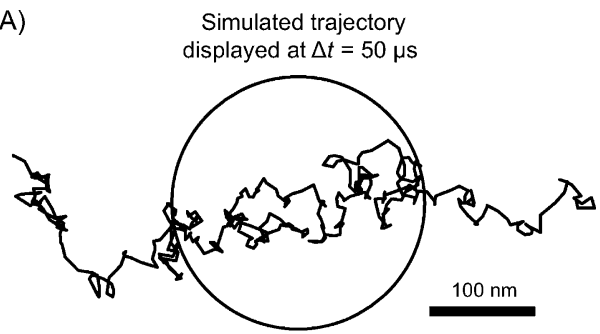

B)

Fast confocalized tracking

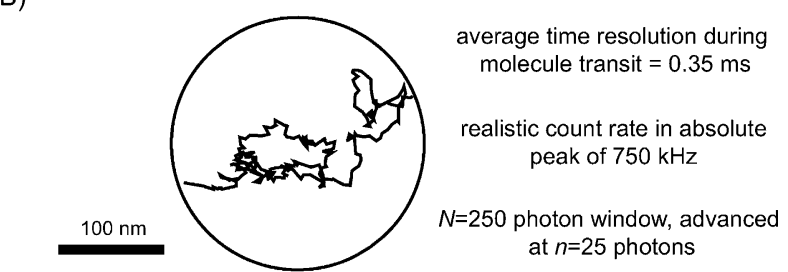

C)

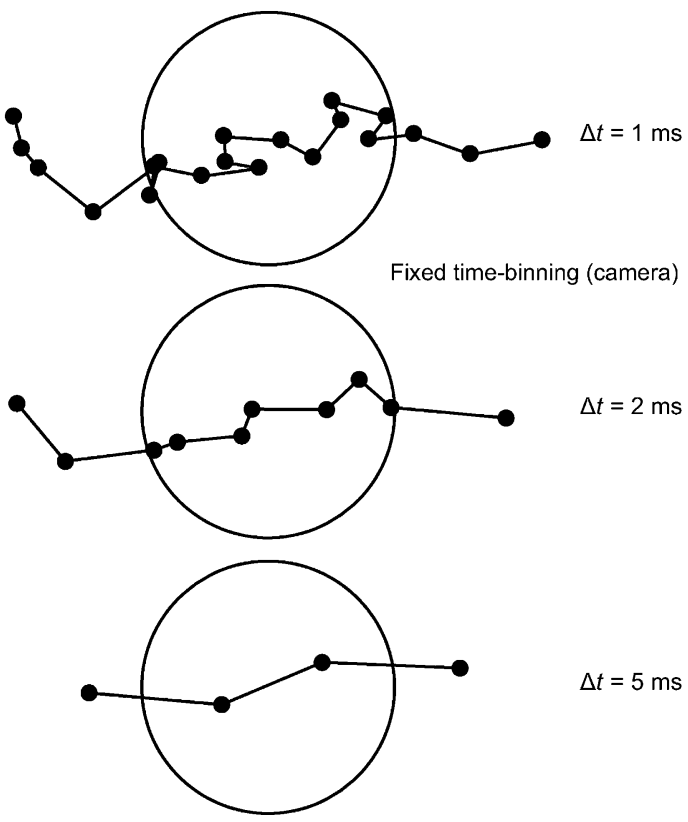

Figure 4. Sampling of the molecular position at finite spatiotemporal resolution. A) Simulated molecule trajectory within a diffraction-limited spot of $250 \mathrm{~nm}$ in diameter. B) Trajectory obtained by fast confocalized tracking, employing the full photon stream. A realistic peak count rate of $750 \mathrm{kHz}$ and an experimental reference map was used in the calculation. C) The trajectories as measured by camera-based tracking at fixed frame times $\Delta t$. Photon collection during the whole frame intervals (i.e. complete time averaging of position) is assumed.

peak count rate from the molecule of $750 \mathrm{kHz}$. (For Atto647N as a label, we frequently observed peak rates of up to $\sim 1.5 \mathrm{MHz}$ ). Figure $4 \mathrm{C}$ gives an indication of the trajectories that would be obtained with camera-based tracking at various integration times $\Delta t$. Camera exposures over 1, 2 or 5 milliseconds (the fastest reported experiments so far have reached $1 \mathrm{~ms}$ for small single-dye experiments) lead to a significant loss of information. While enough photons are collected during these exposure intervals, details of the true trajectory are lost due to time-averaging of the proper motion of the molecule during $\Delta t$. Therefore, the trajectories reflect the center of mass of the true molecule positions during each interval.
Time averaging cannot be avoided in our single-photoncounting approach. However, the time intervals over which we average can be chosen much shorter. Firstly, we are given this opportunity as a result of the high base time resolution of our photon-counting electronics of 50 nanoseconds. Secondly, the peak count rate of $\sim 750 \mathrm{kHz}$ from the single molecule in our setup is high enough to achieve a concomitant high spatial localization precision given by the shot-noise-dominated $\sigma_{r}$ (Figure 2) in less than $0.5 \mathrm{~ms}$. This is because a good fluorophore is intrinsically bright enough to allow-as our experimental scheme shows-more detailed sampling of its path than is achieved by wide-field camera experiments so far. The result is shown in Figure 4B, computed under our typical conditions with an experimental reference map. The obtained trajectory features better sampling than those in Figure $4 \mathrm{C}$ and reproduces the central looping path taken by the molecule. At the periphery of the observation region, the spatiotemporal resolution is lower due to smaller count rates (lower fluorescence excitation and detection efficiency). Therefore, some details in the motion path are lost here. Most importantly, the photon stream readily allows continuous sliding of the integration interval yielding a much finer temporal sampling of the measured trajectory (similar to a moving average). The trajectory displayed in Figure 4B was obtained by shifting a variable time interval comprising a fixed number $N$ of 250 detected photons in increments of $N / 10=25$ photons. One can consider this moving average as being identical to ten independent assessments of one trajectory, each assessment shifted by a tenth of the effective time resolution. This enhanced mode allowed the assembly of histograms of step length $(\Delta r)$ versus time interval $(\Delta t)$ statistics with much higher population than would be found in trajectories sampled "once" (no sliding integration interval). The obtained histograms thus exhibit lower noise (i.e. an enhanced statistical basis) while leaving extracted model parameters unaffected if only positions from non-overlapping time intervals are compared (for implementation details see ref. [32]). Non-overlapping time intervals yield statistically independent positions and warrant that no correlations on short time scales are introduced. In other words, the number of meaningful, statistically independent position pairs for computation of $\left\{\Delta r^{2}, \Delta t\right\}$ is significantly increased using the enhanced (sliding integration interval) mode, bolstering valid statistics. However, and as a matter of course, no time lags less than the time resolution-which is governed by the integration time window, here of size $N$ photons-can independently ever become accessible to the analysis. Thus the sliding integration interval scheme provides for a better use of the full information contained in the photon stream but cannot be regarded as a tool to enhance temporal resolution.

\subsubsection{Short-Term Lateral Confinement}

A focus of the original report of this tracking approach ${ }^{[32]}$ was the demonstration of nanoscale inhomogeneities in the diffusion of membrane-constrained molecules. While the causes of anomalous diffusion may be complex, and indeed there may be more than one responsible mechanism, such anomaly may 
include unambiguous instances of temporary arrest of lateral diffusion. That is, we would like to diagnose instances when the molecule undergoes confinement on the molecular scale, solely on the basis of spatiotemporal trajectories $(x(t), y(t))$. The sliding interval analysis described in the previous section provides better sampling of the start and end times of such trapping events. This is beneficial when trying to identify nanoscale confinement directly from the spatiotemporal trajectories (Figure 5 and ref. [32]).

\subsubsection{Superior Single-Molecule Fluorescence Burst Selection}

The first step of our localization approach is the identification of single-molecule transits through our diffraction-limited observation spot via a burst in the detected signal time trace. Binning groups of fixed photon number in variable time intervals rather than variable photon numbers during a fixed time interval leads to a marked improvement in florescence burst recognition. The fluorescence intensity over time can be calculated on a sliding basis by combining $N$ photons and determining their average arrival time as well as the average count rate during this interval. ${ }^{[32]}$ Figure $6 \mathrm{~A}$ shows that such an analysis effectively flattens the shot-noise-dominated background as compared to the classical time-binned trace (Figure $6 \mathrm{~B}$ ). This is advantageous for a more accurate determination of photon bursts by a threshold criterion.

In many cases, a molecular trajectory thus identified from a single-molecule fluorescence burst (Figure 7) will transiently leave the high-fidelity observation area (the central circle of $250 \mathrm{~nm}$ diameter). Given the prior assumption that the entire burst is caused by one molecule,
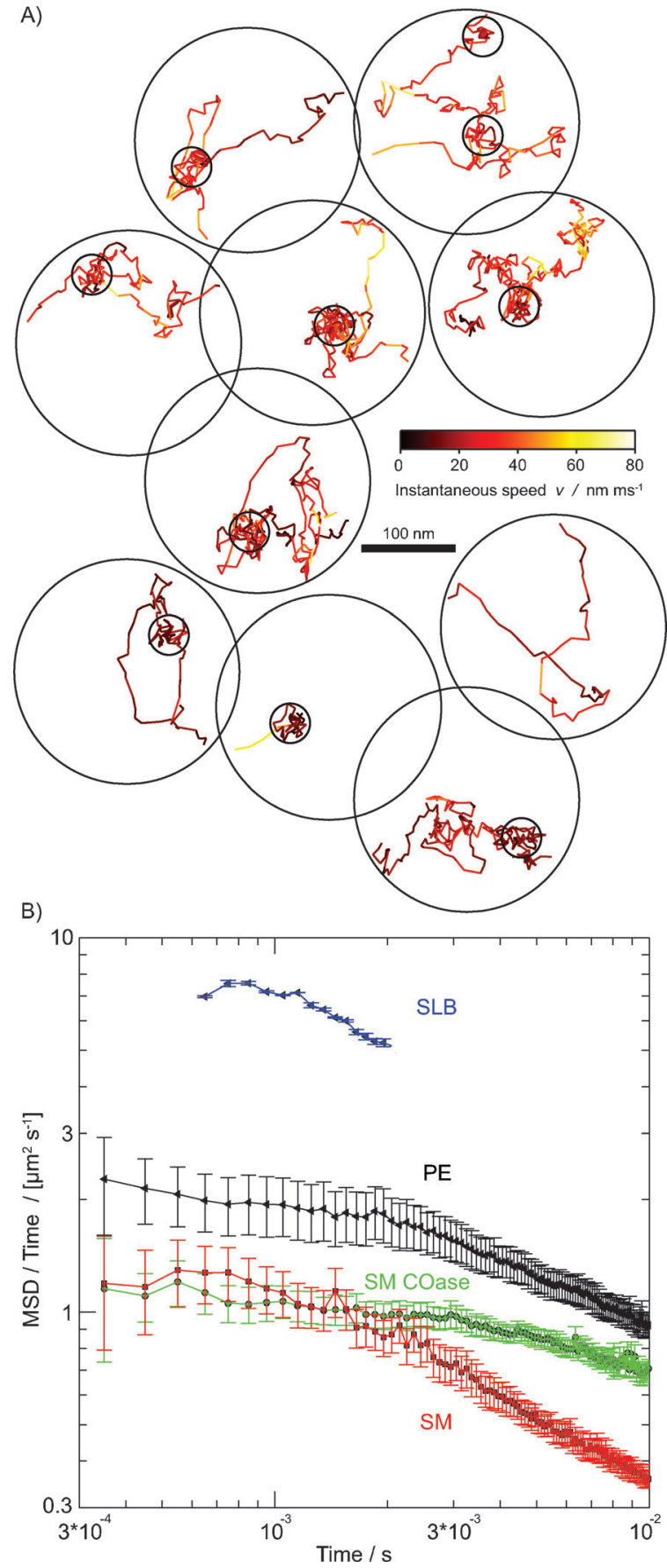

Figure 5. A) Trajectories of a fluorescent sphingomyelin (SM) analog in live mammalian PtK2 cells recorded on the three-detector setup (large black circles: observation area) reveal complex and highly heterogeneous behavior. Hundreds to thousands of trajectories are obtained in a typical 10-minute recording. Their combined analysis allows statistical evaluation and assessment by diffusion models, highlighting for example transient arrests of the SM lipids (small black circles). B) Plot of log(MSD/time) vs. log(time) for tracking experiments ${ }^{\text {[19] }}$ of phosphoethanolamine (PE), sphingomyelin (SM), sphingomyelin after cholesterol depletion of the plasma membrane by cholesterol oxidase (SM COase) and PE diffusing in an artificial supported lipid bilayer on glass (SLB). The localization precision-related offset of $4 \sigma^{2}=4(19 \mathrm{~nm})^{2}=1.44 \times 10^{-3} \mu \mathrm{m}^{2}$ has been subtracted from the MSD. Note the log-log representation. Constant values of MSD/time depict free diffusion, while a decrease indicates hindered diffusion in this time range, in this example due to transient trapping. ${ }^{[32]}$ Note that the very fast diffusion in the SLB even for our approach introduces some bias at longer lag times. Here, the reduction of $\sim 20 \%$ between 1 and 2 ms lag time only apparently signifies a deviation from perfect Brownian diffusion. This artifact stems from the fact that within a millisecond, the fast lipids in the SLB $\left(D>3.5 \mu \mathrm{m}^{2} \mathrm{~s}^{-1}\right)$ already sense the edge of the $250 \mathrm{~nm}$ observation region (and thus an effective confinement to this dimension), an effect which cannot be fully corrected. ${ }^{[32]}$ The diffusion coefficient of the lipid analogs in the live-cell plasma membrane is $D \approx 0.3-0.5 \mu \mathrm{m}^{2} \mathrm{~s}^{-1}$, that is, it takes the lipids on average $20-30 \mathrm{~ms}$ to cross the observation spot. Similar artifacts are therefore potentially introduced only for lag times $>10-20 \mathrm{~ms}$, that is, not within the time range given here. 

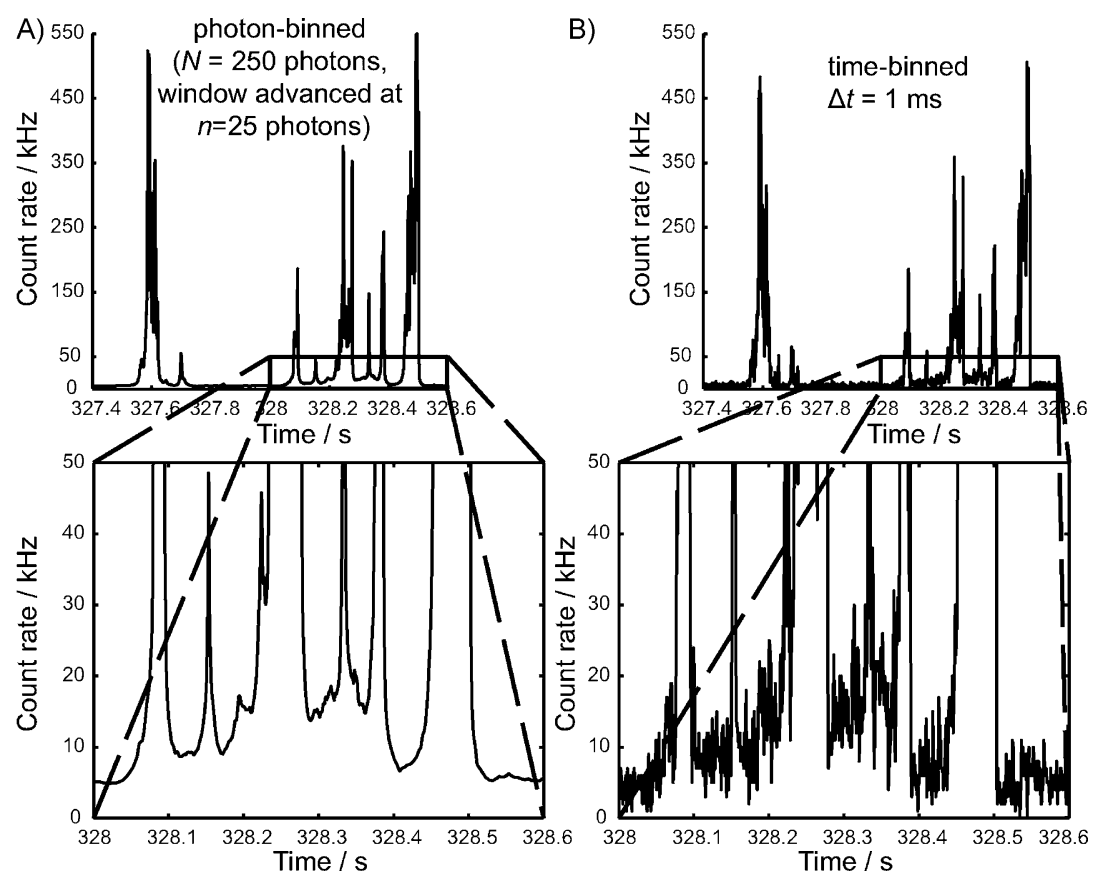

Figure 6. The photon-binned reconstruction of the signal intensity trace $(A)$ features less noise than a trace of the same data binned in fixed time intervals $\Delta t=1 \mathrm{~ms}(\mathrm{~B})$. Photon binning allows a better identification of the start and end times of individual photon bursts. The photon-binned trace was constructed by advancing a window of $N=250$ photons by steps of $n=25$ photons and computing the set $\left\{t_{j}, l_{j}\right\}$ for each window $j$.

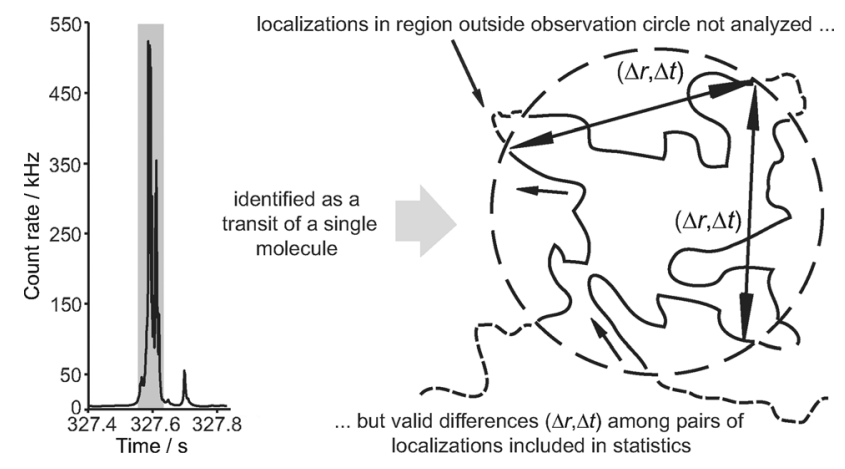

Figure 7. Trajectory analysis for a circular observation area. A molecular trajectory identified from a single-molecule fluorescence burst (left) may reach the border of our observation area (the central circle of $250 \mathrm{~nm}$ diameter), briefly exit from it and return (schematic). Given the prior assumption that the burst contained one molecule and that the molecule has not left the more widely interpreted focus, displacement vectors and longer time lags $(\Delta r, \Delta t)$ to the localizations upon return may still be included in the step length versus lag time statistics.

that is, the molecule has not left the more widely interpreted excitation focus, displacement vectors and longer time lags $(\Delta r, \Delta t)$ between localizations before leaving and after returning to the high-fidelity area may still be included in the step length versus lag time histogram and bolster statistics.

\subsubsection{General Considerations of Spatial versus Temporal Accuracy}

Our method offers especially high flexibility when it comes to choosing appropriate spatial and temporal accuracies to track the molecular motion of interest. Evidently, there are limits to achieving both equally well. Indepth treatments of static and dynamic errors and their consequences in particle tracking experiments may be found in ref. $[34,35]$. When using groups of $N$ photons from our photon stream to yield positions, the effective temporal resolution $\Delta t$, that is, the time interval between two consecutive localizations, is given by $\Delta t \approx N / Q$, with $Q$ the count rate of detected photons. The localization is subject to an uncertainty of $\Delta A \approx$ $A_{\mathrm{PSF}} / N$, where $A_{\mathrm{PSF}}$ is the area of the detection PSF. It follows that $\Delta A \cdot \Delta t \approx A_{\mathrm{PSF}} / Q$. The product of spatial localization precision and temporal resolution can be interpreted as the spatiotemporal resolution of the experiment and is given by the PSF area and the count rate. In our experiments, $\Delta A \cdot \Delta t$ varies slightly over the observation area as the count rate from the molecule is highest at the center of our excitation profile. By choosing an appropriate $N$, it is thus possible to balance the spatial and the temporal resolution as required. Fixing $N$ prioritizes similarity in spatial precision amongst localizations and yields varying time separations, while a fixed integration time $\Delta t$ leads to a variable localization precision depending on the instantaneous count rate. Both approaches must converge to showing essentially the same diffusion properties. However, control over the spatial precision (by fixing $N$ ) has its advantages when studying portions of trajectories where little movement occurs, such as during "trapping". ${ }^{[32]}$ In this particular case, time-averaging shows only negligible influence. For example, we have previously used low fixed $N$ and thus short (varying) integration times $\Delta t$ to identify pausing or traps within tracks of fluorescent lipid analogs in the plasma membrane of living cells, and re-analyzed the movement within these traps with large $N$ to validate that the lipids where hardly moving during trapping. ${ }^{[32]}$ The choice of effective temporal sampling, given by the number of photons $N$ per localization estimate, should depend on the diffusion coefficient $D$ and the count rate $Q$ of a measurement situation. Motion of the molecule during the signal integration period $\Delta t=N / Q$ adds a "motion blur" to the positional uncertainty from photon shot noise. In a simplified view ignoring motion other than diffusion, this may be described by addition of errors in quadrature [Eq. (6)]:

$\sigma_{\text {effective }}^{2} \approx \frac{\sigma_{\mathrm{PSF}}^{2}}{N}+4 D \frac{N}{Q}$ 
where the first term describes the familiar error-in-mean improvement in the localization estimate based on $N$ recorded photons, and the second term describes the area covered by the molecule during a diffusional process (random walk) within the time interval $\Delta t$. The above expression is formulated in analogy with the argument by areas [Eq. (7)]:

$\Delta A_{\text {total }} \approx \frac{A_{\mathrm{PSF}}}{N}+\pi 4 D \Delta t=\frac{A_{\mathrm{PSF}}}{N}+\pi 4 D \frac{N}{Q}$

Consequently, $\sigma_{\text {effective }}$ has a global minimum for [Eq. (8)]:

$N=\left(\frac{Q}{4 D}\right)^{1 / 2} \sigma_{\mathrm{PSF}}$

guiding our choice of $N$ in the described experiments. Evidently, for increasing $N$ (combining photons over a longer time) and $D$ (molecules diffuse faster), the "motion blur" becomes more significant. Hence, for fast-diffusing molecules a smaller $N$ should be chosen.

\subsection{Full Photon Stream for Imaging-Optical Nanoscopy (GSDIM)}

In the following we depict how our optical setup can-in addition to the recording of single-molecule tracks-(simultaneously) resolve cellular structures with a spatial resolution below the diffraction limit of conventional optical microscopes, at least on a $<1 \mu \mathrm{m}^{2}$ field of view. Specifically, we show how the information of precise localization of individual molecules in the focal plane (by combining photon count information on distinct detectors) can be used to obtain sub-diffraction spatial information.

Diffraction of light poses a major limitation on optical farfield microscopy, since the focusing of light impedes the distinction of alike objects that are closer together than approximately half the wavelength of light, that is, about $200 \mathrm{~nm}$ for visible light. ${ }^{[36]}$ As a consequence, details of cellular structures on smaller spatial scales cannot be resolved properly. Recently developed optical super-resolution microscopes or nanoscopes overcome this limitation by ensuring that fluorescence is emitted from a single feature at a time only. ${ }^{[37]}$ Specifically, all optical nanoscopy approaches make use of (optically induced) transitions between states of the fluorescent label with different emission characteristics (such as a bright and dark state). For example, in stimulated emission depletion microscopy $(\mathrm{STED})^{[38,39]}$ emission is only allowed from fluorophores located in sub-diffraction sized areas defined by laser light featuring one or several intensity zeros at the sample. On the other hand, techniques such as (f)PALM or (d)STORM implement transitions between on- and dark off-states stochastically in space, on a single-molecule basis so to ensure that fluorescence emission is at a time occurring from individual molecules only, which are then further apart from each other than the $200 \mathrm{~nm}$ diffraction limit. ${ }^{[40-42]}$ Imaging of their fluorescence emission onto a camera enables localization of their spatial po- sitions with $\mathrm{nm}$ precision, and on- and off-switching of different isolated molecules in subsequent camera frames allows the reconstruction of an image with sub-diffraction resolution. In GSDIM (or (d)STORM or blinking microscopy) transitions between on- and off-states are stochastically induced by transient shelving of the majority of molecules into metastable dark states (on-off blinking). ${ }^{[43-46]}$

Here, fluorescent molecules within a $\sim \varnothing 5 \mu$ m (FWHM) excitation focus were subjected to blue light $(\lambda=491 \mathrm{~nm})$ by a laser source (Calypso, Cobolt) at intensities of $\sim 5-10 \mathrm{~kW} \mathrm{~cm}^{-2}$. This resulted in the on- and off-blinking of the irradiated fluorescent molecules. To allow the simultaneous emission of a single molecule within this area only, we had to realize long off-periods of our labels. Two methods for increasing the lifetime of the dark states beyond the millisecond range were applied in the present work. Oxygen reduction leads to long lifetimes of dark states such as triplet or redox states populated thereof, since oxygen serves as a triplet quencher. Therefore, aqueous media with addition of glucose, glucose-oxidase and catalase were used. Glucose-oxidase is an enzyme which uses glucose to bind oxygen, whereas catalase is an enzyme which decomposes the resulting toxic hydrogen peroxide. Alternatively, the samples were embedded in poly-vinyl-alcohol (PVA). PVA constrains the oxygen diffusion which leads to a prolonged triplet lifetime and furthermore introduces additional dark states. ${ }^{[4]}$ Both media are standard microscopy mounting media.

The fluorescence emission of a single molecule in the $\varnothing 5 \mu \mathrm{m}$ (FWHM) excitation area was imaged onto a fiber bundle of approximately $2 \times 2 \mu \mathrm{m}^{2}$ in size when projected onto the sample plane (magnification $\sim 200$ as before). Instead of the aforementioned three fibers, our detection assembly was enhanced with seven fiber inputs, each serving a separate singlephoton counting detector, and each collecting light from a slightly different part of the excitation area (Figure $8 \mathrm{~A}-\mathrm{C}$ ). We now did not determine the spatiotemporal track of the single molecule, but only had to determine its spatial position from the photons detected during its on-phase. This localization was performed in the way as before, that is by comparing the set of the signals detected on all detectors to a calibration set (Figure 8D,E). Once the emissions of individual emitters were temporally separated (unmixed, as outlined later on), the resolution of the resulting image reconstructed from many such localizations is determined by the localization precision for individual molecules. ${ }^{[3,46]}$ While the outer region-where the signal is largely collected in just one fiber, rendering the identification of position very imprecise because of the high relative noise in the other fiber detectors-should be excluded, Figure $8 \mathrm{~F}$ and $\mathrm{G}$ show that positions in the central region of approximately $\varnothing 500-600 \mathrm{~nm}$ were reproduced with high fidelity. In this central region (marked in white in Figure $8 \mathrm{G}$ ), the localization precision was largely governed by the shot noise limit and followed the expected dependence on the detected number of photons $N$ shown in Figure $8 \mathrm{~F}$. A fit of [Eq. (9)]:

$\sigma_{\text {eff }}=\left(\sigma_{\text {shotnoise }}^{2}+\sigma_{\text {vib }, \text { sys }}^{2}\right)^{1 / 2}=\left(\frac{A^{2}}{N}+\sigma_{\text {vib }, \text { sys }}^{2}\right)^{1 / 2}$ 
to the data resulted in the values $A \approx 262 \mathrm{~nm}$ for the FWHM diameter of the detection PSF and $\sigma_{\text {vib,sys }} \approx 7 \mathrm{~nm}$ for the localization uncertainty due to imperfections such as setup instability (vibrations), systematic errors due to finite pixel size in the reference map or unfavorable noise from further-away detectors.

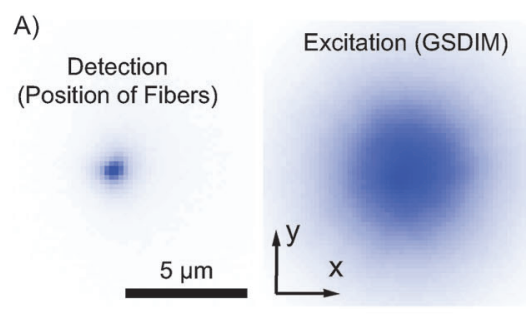

B)

Fiber input faces $1-7$

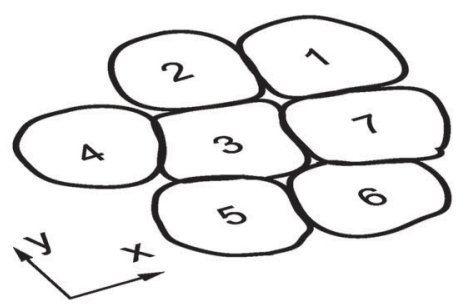

D)

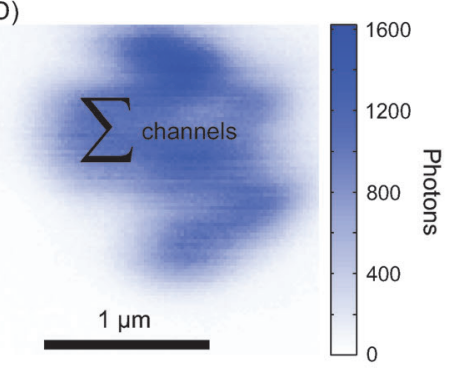

F)

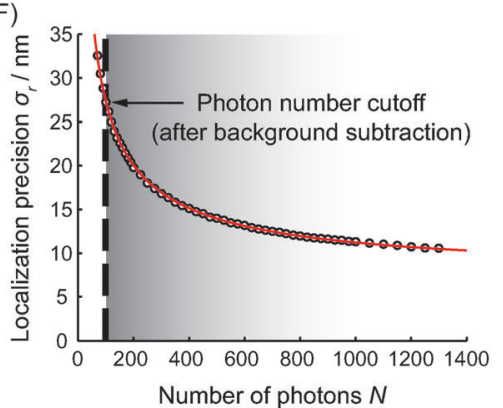

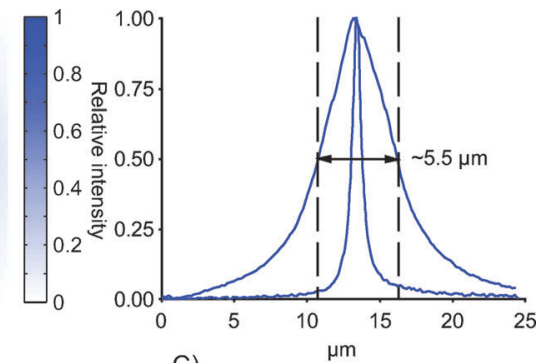

C)

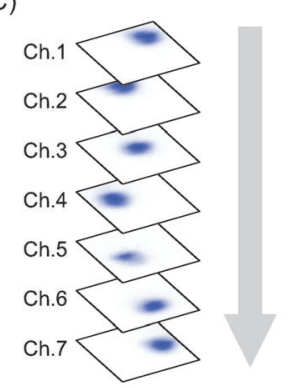

E)

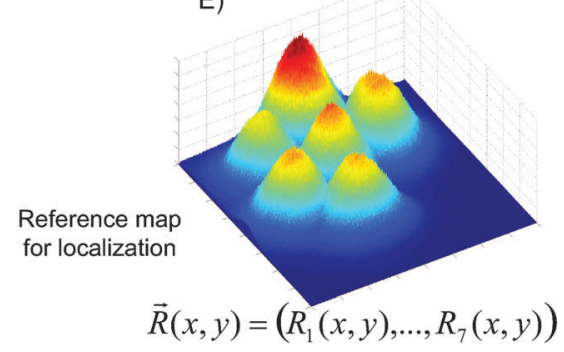

G)

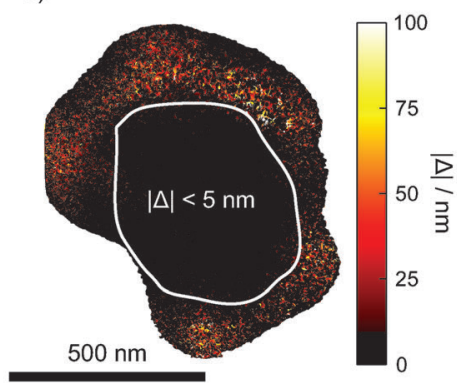

Figure 8. Proof-of-principle experiments with the "single photon camera". A) To ensure the efficient transfer of a majority of molecules to non-fluorescent dark states over a larger region, a $\varnothing 5 \mu \mathrm{m}$ (FWHM) excitation focus was created (middle), centered on the position of the fiber bundle for detection [much smaller area (left), fluorescence recorded from a dilute fluorophore solution]. Line profiles show how the fiber collection position coincides with the pump (excitation) light (right). B) The seven-fiber "single photon camera" detector elements. C) Signals from a fluorescent bead are recorded on all seven channels as a function of position $(x, y)$ (scanned area:

$2 \times 2 \mu \mathrm{m}^{2}$ ). D) The sum of counts on all seven detectors [as measured in (C)] varies smoothly over the recorded region, but $\mathrm{E}$ ) the ratios among detectors uniquely identify a given localization by its count pattern

$\vec{R}(x, y)=\left(R_{1}(x, y), \ldots, R_{7}(x, y)\right)$ (the reference map), as can be seen from the individual responses of the detection elements (signal "mountains"), which decay away from the fiber centers as other fibers pick up more signal.

F) The localization precision $\sigma_{\mathrm{r}}$ versus photons $N$ employed in the localization is nearly shot noise limited as determined by 5000 consecutive localizations of a stationary bead in the central region. These values imply a lower bound on the attainable precision and thus spatial resolution in the image for respective photon numbers in a typical emission event. G) The map self-consistency check (see Supporting Information of ref. [32]) shows that localization is reliable (no systematic errors) over the circumscribed central region of about 500-600 nm diameter.
Most likely owing to the last influence (noise content only in detectors remote from the imaged lateral emitter position), this value was worse than for the molecule tracking configuration, where we operated only in the region directly between three fibers $\left(\sigma_{\text {vib,sys }}<2 \mathrm{~nm}\right)$. Over the central region the setup therefore allowed near-shotnoise-limited localization based on spatially resolved single photon counting.

To exemplify GSDIM nanoscopy with our seven-detector setup, the microtubule network of whole fixed PtK2 cells was immunostained with Alexa488. The mammalian PtK2 cell line was grown as described in ref. [48]. The cells were then seeded and grown on standard glass coverslips to a confluency of about $50 \%$ and fixed with cold methanol for $4 \mathrm{~min}$, followed by incubation in blocking buffer [phosphate buffered saline (PBS) containing $1 \%$ bovine serum albu$\min$ (BSA)]. The microtubules were stained with anti- $\beta$-tubulin mouse lgG (Sigma-Aldrich, St. Louis, MO, USA) and the dye Alexa488 conjugated to sheep anti-mouse IgG (Sigma). Imaging of the immunostained cells was performed in PVA [spincoating of $1 \%$ PVA in PBS (pH 7.4) solution at $3000 \mathrm{rpm}$ for $30 \mathrm{~s}]$. Photon streams were recorded for several minutes on seven detectors. One fiber had low transmission but was included in the signal analysis. From the combined photon trace over all seven detectors, individual fluorescence bursts could be identified-now corresponding not to diffusion transits but to onphases of individual fluorophores (Figure 9A). A recording mode with a fixed integration time (e.g. a camera) cannot adequately match the duration of individual bursts and inevitably comprises periods devoid of molecular signal during a frame (Figure 9B). The single-photonbased burst selection can be limited to the photons stemming only from the time interval of the emission event. Thus virtual- 

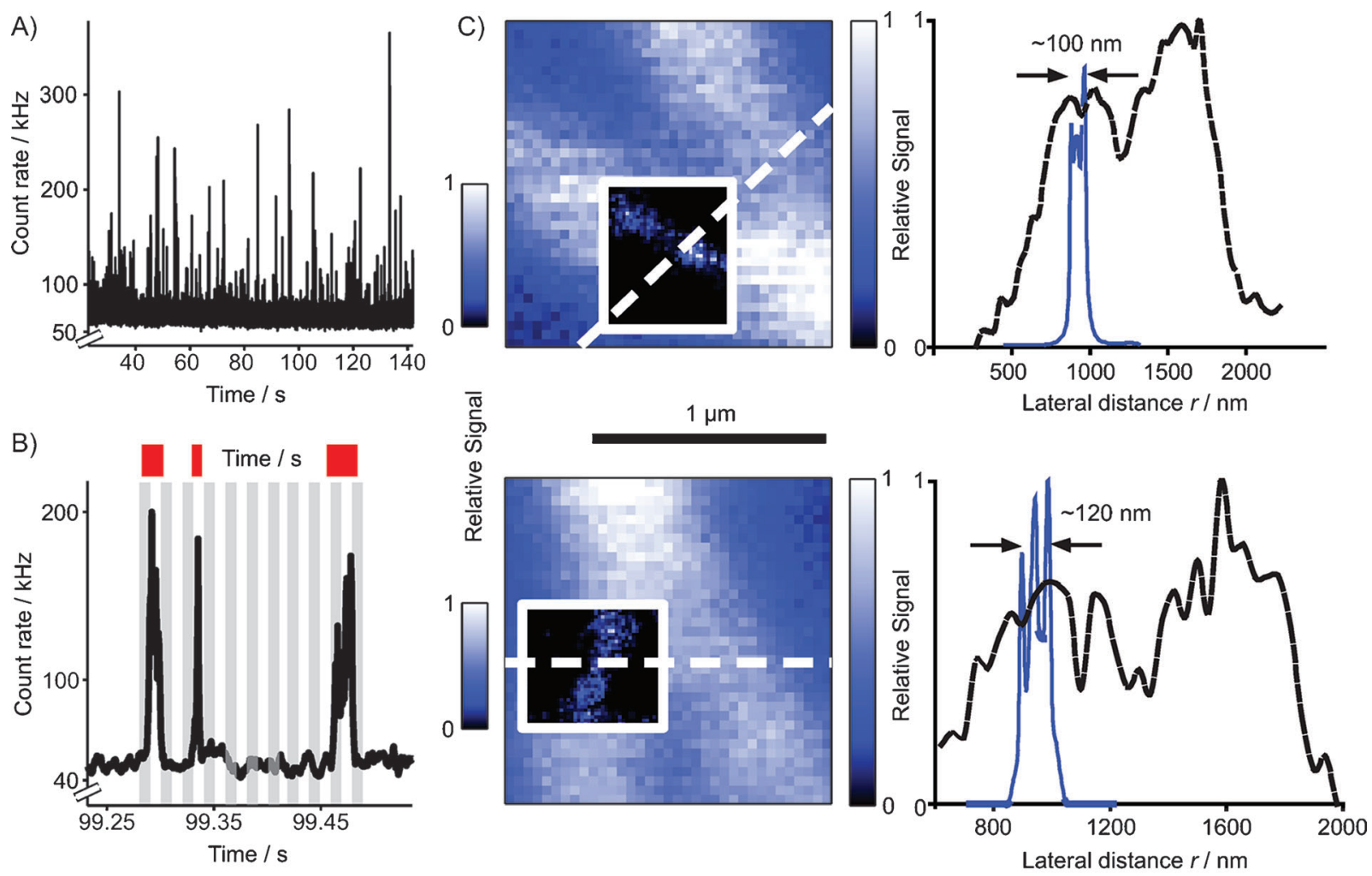

Tand $\Delta t=10 \mathrm{~ms}$ fixed bins photonstream-

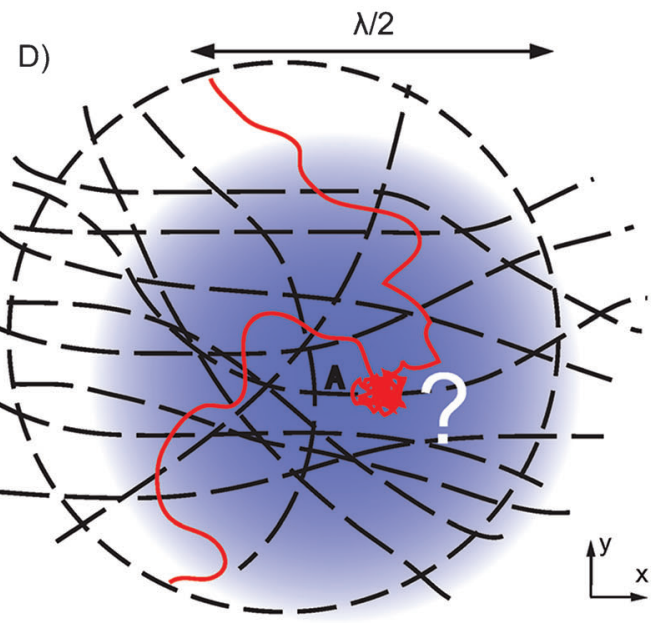

Fast molecular tracking

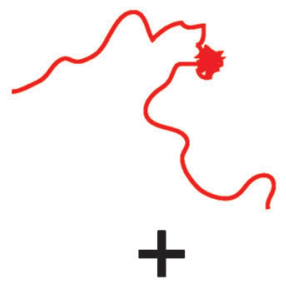

GSDIM, PALM, STORM nanoscopy of membrane skeleton

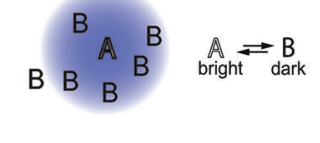

Figure 9. GSDIM nanoscopy. Assessment of the burst selection based on complete photon statistics. A) Count rate over time (photon-binned). Individual peaks in signal correspond to photon bursts of individual molecules in the on-state. They are rare enough so as not to overlap in time. B) Fluorescence bursts of variable duration from individual emitting molecules can be accurately identified from a photon-binned intensity trace. Time binning (grey, shown for $\Delta t=10 \mathrm{~ms}$ ) inevitably combines bursts occasionally (or cuts them in half) since the exact burst duration is a stochastic outcome and the bin intervals are fixed. C) Examples of GSDIM nanoscopy with the seven-fiber "single-photon camera" (white box, field of view Ø500-600 nm). The ratios among the seven detection channels uniquely determine each emitter's position. Two examples of imaged portions of the microtubular network (ß-tubulin) in whole fixed PtK2 cells are shown. $\beta$-tubulin was visualized by indirect immunolabeling with an Alexa488 secondary antibody and embedding in polyvinyl alcohol (PVA) before imaging. Line profiles across strands (right) suggest an image resolution of $\sim 60-70 \mathrm{~nm}$ (FWHM) in agreement with the photon cutoff of $N=100$ photons (antibody-labeled microtubules are $\geq 60 \mathrm{~nm}$ in diameter). The confocal scan over $1.5 \times 1.5 \mu \mathrm{m}^{2}$ for comparison has a resolution of about $250 \mathrm{~nm}$. The scale bar is $1 \mu \mathrm{m}$. (D) GSDIM nanoscopy paired with fast molecular tracking (schematic trajectory in red) could provide a structural context of trapping at the nanoscale. While one color channel records molecular trajectories, this spatiotemporal information could be correlated with nanoscale structural information about the cellular context (such as location of the membrane skeleton, dotted lines), generated in the other channel (by PALM, STORM, GSDIM nanoscopy etc.). The lower right sketch exemplifies the principle of the GSDIM nanoscopy approach. The fluorescent labels are stochastically transferred between a bright A and a dark B state, thereby leaving only one molecule at a time in the bright state A within the diffraction-limited observation spot (blue circle). 
ly none of the avoidable background periods are contained in the chosen time segments for localizations.

Figure $9 \mathrm{C}$ shows examples of single-photon-counting GSDIM images (insets) of tubulin strand segments as compared to a quasi-confocal scan with one of the detection fibers. A resolution of $\mathrm{FWHM} \approx 60 \mathrm{~nm}$ is estimated from the shown line profiles, in accordance with the threshold on the photon number $N \geq 100$ employed, which should guarantee a localization accuracy of that order over the small central region. Images produced by localizing events from the same photon stream based on time bins of 5 and $10 \mathrm{~ms}$, respectively, exhibit effectively the same width within errors (not shown). Frame times of 5 or $10 \mathrm{~ms}$ would be chosen for a camera recording to closely match the characteristic burst times $\Delta t_{\text {burst,ch }} \approx 2.5 \mathrm{~ms}$ extending to several milliseconds. More uncertainty should conceptually be introduced in individual localizations for the latter cases as a result of the unwanted background periods, slightly reducing the resolution in the images. The last presumption will need further testing and theoretical analysis, since background is always present to varying degrees in cellular imaging applications. In principle, reducing the time-integrated periods of acquired background should improve localization precision and image resolution.

\section{Conclusions}

The technology in image sensors such as electron-multiplying charge-coupled devices (EMCCDs) and scientific complementary metal-oxide semiconductor (sCMOS) cameras advances rapidly and has closed the gap to millisecond acquisition over large fields of view at high quantum yield. ${ }^{[49]}$ However, singlephoton counting APDs possess excellent noise characteristics and deliver a photon stream which proved very beneficial in this fast molecular tracking implementation. Our proof-of-principle experiments suggest that high-speed tracking may be compatible with simultaneous or interleaved imaging of a quasi-static molecular assembly in another color channel (Figure 9D). This capability may in future shed light on any hitherto unknown determinants of molecular trapping instances, such as underlying cytoskeletal protein assemblies.

\section{Acknowledgements}

We thank Dr. Alf Honigmann and Dr. Veronika Mueller for helpful discussions, Dr. Michael Hilbert for help with the setup, as well as Dr. Ellen Rothermel and Tanja Gilat for excellent technical assistance (all MPI for Biophysical Chemistry). This work was supported through a Leibniz Research Award from the Deutsche Forschungsgemeinschaft to S.W.H., and the DFG-funded SFB755 to S.W.H. and C.E.

Keywords: confocal microscopy • lipid dynamics · nanoscopy • single molecule microscopy $\cdot$ super-resolution

[1] J. R. Lakowicz, Principles of Fluorescence Spectroscopy, 3rd ed., Springer, New York, 2006.
[2] J. Lippincott-Schwartz, E. Snapp, A. Kenworthy, Nat. Rev. Mol. Cell Biol. 2001, 2, 444-456.

[3] D. Axelrod, D. Koppel, J. Schlessinger, E. Elson, W. Webb, Biophys. J. 1976, 16, 1055-1069.

[4] N. L. Thompson, T. P. Burghardt, D. Axelrod, Biophys. J. 1981, 33, 435454.

[5] C. A. Day, L. J. Kraft, M. Kang, A. K. Kenworthy, Curr. Protein Cytometry 2012, 2-19.

[6] D. Magde, E. L. Elson, W. W. Webb, Biopolymers 1974, 13, 29-61.

[7] E. Haustein, P. Schwille, Annu. Rev. Biophys. Biomol. Struct. 2007, 36, $151-169$.

[8] Z. Petrasek, J. Ries, P. Schwille, Methods Enzymol. 2010, 472, 317-343.

[9] M. A. Digman, E. Gratton, Annu. Rev. Phys. Chem. 2011, 62, 645-668.

[10] P. F. Lenne, L. Wawrezinieck, F. Conchonaud, O. Wurtz, A. Boned, X. J. Guo, H. Rigneault, H. T. He, D. Marguet, EMBO J. 2006, 25, 3245-3256.

[11] D. L. Kolin, P. W. Wiseman, Cell Biochem. Biophys. 2007, 49, 141-164.

[12] L. Kastrup, H. Blom, C. Eggeling, S. W. Hell, Phys. Rev. Lett. 2005, 94, 178104.

[13] C. Eggeling, C. Ringemann, R. Medda, G. Schwarzmann, K. Sandhoff, S. Polyakova, V. N. Belov, B. Hein, C. von Middendorff, A. Schönle, S. W. Hell, Nature 2009, 457, 1159-1163.

[14] C. Ringemann, B. Harke, C. von Middendorff, R. Medda, A. Honigmann, R. Wagner, M. Leutenegger, A. Schönle, S. W. Hell, C. Eggeling, New J. Phys. 2009, 11, 103054.

[15] V. Mueller, C. Ringemann, A. Honigmann, G. Schwarzmann, R. Medda, M. Leutenegger, S. Polyakova, V. N. Belov, S. W. Hell, C. Eggeling, Biophys. J. 2011, 101, 1651-1660.

[16] A. Honigmann, V. Mueller, S. W. Hell, C. Eggeling, Faraday Discuss. 2013, $161,77-89$

[17] F. Göttfert, C. A. Wurm, V. Mueller, S. Berning, V. C. Cordes, A. Honigmann, S. W. Hell, Biophys. J. 2013, 105, L01-L03.

[18] K. Simons, M. J. Gerl, Nat. Rev. Mol. Cell Biol. 2010, 11, 688-699.

[19] T. Fujiwara, K. Ritchie, H. Murakoshi, K. Jacobson, A. Kusumi, J. Cell Biol. 2002, 157, $1071-1081$.

[20] A. Kusumi, C. Nakada, K. Ritchie, K. Murase, K. Suzuki, H. Murakoshi, R. S. Kasai, J. Kondo, T. Fujiwara, Annu. Rev. Biophys. Biomol. Struct. 2005, 34, $351-378$.

[21] M. L. Kraft, Mol. Biol. Cell 2013, 24, 2765-2768.

[22] H. Geerts, Biophys. J. 1987, 52, 775-782.

[23] M. P. Sheetz, S. Turney, H. Qian, E. L. Elson, Nature 1989, 340, 284-288.

[24] A. Yildiz, J. N. Forkey, S. A. McKinney, T. Ha, Y. E. Goldman, P. R. Selvin, Science 2003, 300, 2061-2065.

[25] M. Vrljic, S. Y. Nishimura, S. Brasselet, W. E. Moerner, H. M. McConnell, Biophys. J. 2002, 83, 2681-2692.

[26] M. Vrljic, S. Y. Nishimura, W. E. Moerner, H. M. McConnell, Biophys. J. 2005, 88, 334-347.

[27] S. Wieser, M. Moertelmaier, E. Fuertbauer, H. Stockinger, G. Schütz, Biophys. J. 2007, 92, 3719-3728.

[28] K. Jaqaman, H. Kuwata, N. Touret, R. Collins, W. S. Trimble, G. Danuser, S. Grinstein, Cell 2011, 146, 593-606.

[29] M. Clausen, B. C. Lagerholm, Curr. Protein Pept. Sci. 2011, 12, 699-713.

[30] N. Bobroff, Rev. Sci. Instrum. 1986, 57, 1152-1157.

[31] R. E. Thompson, D. R. Larson, W. W. Webb, Biophys. J. 2002, 82, $2775-$ 2783.

[32] S. J. Sahl, M. Leutenegger, M. Hilbert, S. W. Hell, C. Eggeling, Proc. Natl. Acad. Sci. USA 2010, 107, 6829-6834.

[33] M. Leutenegger, R. Rao, R. A. Leitgeb, T. Lasser, Opt. Express 2006, 14, $11277-11291$.

[34] D. S. Martin, M. B. Forstner, J. A. Käs, Biophys. J. 2002, 83, 2109-2117.

[35] T. Savin, P. S. Doyle, Biophys. J. 2005, 88, 623-638.

[36] E. Abbe, Arch. Mikrosk. Anat. 1873, 9, 413-468.

[37] S. W. Hell, Nat. Methods 2009, 6, 24-32.

[38] S. W. Hell, J. Wichmann, Opt. Lett. 1994, 19, 780-782.

[39] T. A. Klar, S. Jakobs, M. Dyba, A. Egner, S. W. Hell, Proc. Natl. Acad. Sci. USA 2000, 97, 8206-8210.

[40] E. Betzig, G. H. Patterson, R. Sougrat, O. W. Lindwasser, S. Olenych, J. S. Bonifacino, M. W. Davidson, J. Lippincott-Schwartz, H. F. Hess, Science 2006, 313, 1642-1645.

[41] S. T. Hess, T. P. K. Girirajan, M. D. Mason, Biophys. J. 2006, 91, 42584272.

[42] M. J. Rust, M. Bates, X. Zhuang, Nat. Methods 2006, 3, 793-796. 
[43] J. Fölling, M. Bossi, H. Bock, R. Medda, C. A. Wurm, B. Hein, S. Jakobs, C. Eggeling, S. W. Hell, Nat. Methods 2008, 5, 943-945.

[44] M. Heilemann, S. van de Linde, A. Mukherjee, M. Sauer, Angew. Chem. 2009, 121, 7036-7041; Angew. Chem. Int. Ed. 2009, 48, 6903-6908.

[45] C. Steinhauer, C. Forthmann, J. Vogelsang, P. Tinnefeld, J. Am. Chem. Soc. 2008, 130, 16840-16841.

[46] S. J. Sahl, W. E. Moerner, Curr. Opin. Struct. Biol. 2013, 23, 778-787.

[47] R. Zondervan, F. Kulzer, S. B. Orlinskii, M. Orrit, J. Phys. Chem. A 2003, $107,6770-6776$.
[48] K. Weber, T. Bibring, M. Osborn, Exp. Cell Res. 1975, 95, 111-120.

[49] F. Huang, T. M. P. Hartwich, F. E. Rivera-Molina, Y. Lin, W. C. Duim, J. J. Long, P. D. Uchil, J. R. Myers, M. A. Baird, W. Mothes, M. W. Davidson, D. Toomre, J. Bewersdorf, Nat. Methods 2013, 10, 653-658.

Received: November 19, 2013 